\title{
Successful en bloc resection for femoral head clear cell chondrosarcoma without biopsy: A case report
}

\author{
MANABU HOSHI, NAOTO OEBISU, YOICHI OHTA, AYAKA TOMIMOTO and HIROAKI NAKAMURA \\ Department of Orthopedic Surgery, Osaka City University Graduate School of Medicine, Osaka 545-8585, Japan
}

Received August 31, 2021; Accepted October 14, 2021

DOI: $10.3892 / \mathrm{mco} .2021 .2459$

\begin{abstract}
Clear cell chondrosarcoma (CCCS) is a rare, low-grade, malignant chondrogenic bone tumour. This tumour commonly affects the epiphysis of long bones, particularly in the proximal femur. The present study reported on the case of a 58-year-old male with right hip pain of five months duration. Plain radiography, magnetic resonance imaging and computed tomography scan revealed the characteristic appearance of chondroid mineralisation in the right femoral head, suggesting typical CCCS. Although biopsy is the gold standard for definite diagnosis prior to treatment, wide resection with removal of the biopsy tract is thought to negatively affect the surgical margin and postoperative hip function. Therefore, en bloc resection without any biopsy and a hip hemiarthroplasty were performed. The pathological diagnosis was CCCS and an adequate surgical margin was obtained. No local recurrence or distant metastases were detected and postoperative function was excellent at the final follow-up. The femoral head is a typical location of CCCS. Wide resection with adequate margins is the main treatment strategy for CCCS and when radiological features are typical, en bloc resection without a biopsy is an acceptable treatment option to improve patient outcomes.
\end{abstract}

\section{Background}

Clear cell chondrosarcoma (CCCS) was first described as a clear cell variant of chondrosarcoma by Unni et al (1). CCCS is rare, accounting for only $2.5-2.7 \%$ of all chondrosarcomas $(2,3)$. CCCS more frequently affects males and is widely distributed across different age groups (4). This lesion characteristically appears at the epiphyses of long bones, particularly in the proximal femur and humerus (5).

Correspondence to: Dr Manabu Hoshi, Department of Orthopedic Surgery, Osaka City University Graduate School of Medicine, 1-4-3 Asahi-Machi, Abeno-ku, Osaka 545-8585, Japan

E-mail: hoshi@med.osaka-cu.ac.jp

Abbreviation: CCCS, clear cell chondrosarcoma

Key words: biopsy, clear cell chondrosarcoma, biopsy, en bloc resection, femur head
Radiological and pathological diagnoses are challenging due to the rarity of the condition and this may commonly lead to inadequate surgical treatment. Patients undergoing initial curettage reportedly had a high local recurrence rate; however, patients who underwent wide resection had a low recurrence rate (4). The initial surgical margin for CCCS was observed to correlate with the oncological outcome (5).

Biopsy has been the gold standard for diagnosing and managing malignant bone tumours. Unfortunately, tumour cell seeding in the biopsy tract is a risk factor for local recurrence. Therefore, biopsy tract removal is widely accepted (6). This frequently requires sacrificing more healthy tissues and may also lead to poor postoperative limb function.

The present study reports on a typical case of CCCS in the right femoral head that was highly suspected using only radiological methods. The patient was successfully treated with an en bloc resection without requiring a biopsy of the lesion.

\section{Case presentation}

A 58-year-old male patient reported right hip pain from June 2018. The patient had visited a nearby clinic and an abnormality had been observed in the right femoral head on a plain radiograph. The patient was then referred to Osaka City University Hospital (Osaka, Japan) in November 2018. Plain radiography revealed fuzzy, irregular calcifications on the femoral head with an indistinct distal margin (Fig. 1). Magnetic resonance imaging (MRI) was performed with iso signal intensity on the T1-weighted image (T1WI; Fig. 2A) and on T2WI, heterogeneously high signal intensity with a focal enhancement of spotty areas was observed (Fig. 2B). The lesion was peripherally enhanced with contrast material on the T1 fat-suppressed image. The decreased spotty signal intensity in central areas revealed matrix mineralisation (Fig. 2C). A computed tomography (CT) scan also revealed a honeycomb-like calcification on the femoral head (Fig. 2D). No cortical destruction was observed. The tumour size was 40x30x42 mm. At this point, CCCS was highly suspected in the patient.

The hip pain was persistent and surgical treatment was proposed to the patient. En bloc resection and a hip hemiarthroplasty (Fig. 3) were performed through an invasive mini-incision anterolateral approach to detach the anterior third of the gluteus medius to expose the hip joint (7). At two days after the surgery, the patient was able to walk with a walker aid. There were no adverse effects of surgical site 


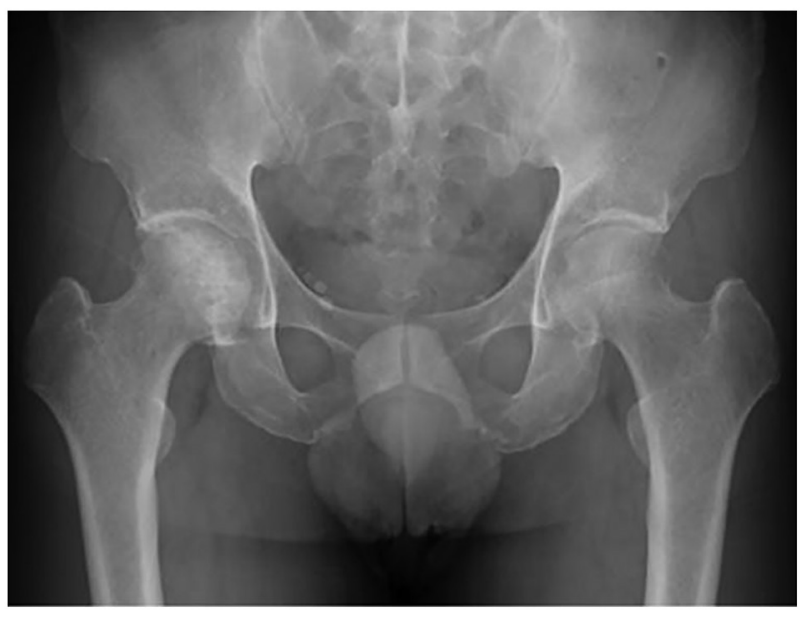

Figure 1. Plain radiography revealing an osteolytic lesion with chondroid matrix mineralisation in the right femoral head.

infection and hip dislocation in his clinical course. The gross findings of the resected femoral head tumour indicated a mixture of white and yellow lesions with well-defined borders. Chondroid tissue and ossification were also visible. The surface cartilage of the femoral head was smooth and intact, without any evidence of a pathological fracture (Fig. 4A). Microscopic examination of the resected specimen indicated that the tumour was composed of a sheet-like proliferation of atypical cells with abundant clear cytoplasm, swollen nuclei and scattered bone formation (Fig. 4B). These pathological diagnoses were consistent with the typical presentation of CCCS. A 2-cm-wide margin was obtained after evaluating the site according to the Japanese Orthopaedic Association evaluation system (8).

At the final follow-up at 32 months after surgery (7), no local recurrence or metastases were observed. The postoperative functional outcome was measured using the International Symposium on Limb Salvage score (9). The patient scored 30 points, which is the highest possible outcome.

\section{Discussion}

In the current case, radiological imaging with plain radiography, MRI and CT scan revealed a typical presentation of CCCS in the right femoral head. Therefore, an incisional biopsy was deemed unnecessary and an en bloc resection and hip hemiarthroplasty were performed without any preoperative biopsy. Pathological diagnosis confirmed the presence of CCCS and a clear surgical margin was achieved. The postoperative limb function of the patient was excellent at the final follow-up.

In cases of a suspicious malignant bone tumour located in the femoral head, incisional biopsy is planned from the lateral side of the proximal femur through the femoral neck to prevent the tumour from contaminating the hip joint space (10). To remove a biopsy tract that potentially contains tumour cells, hip replacement with a tumour prosthesis is necessary after en bloc resection, resulting in poor postoperative function compared to that achieved through hip hemiarthroplasty. Therefore, after considering
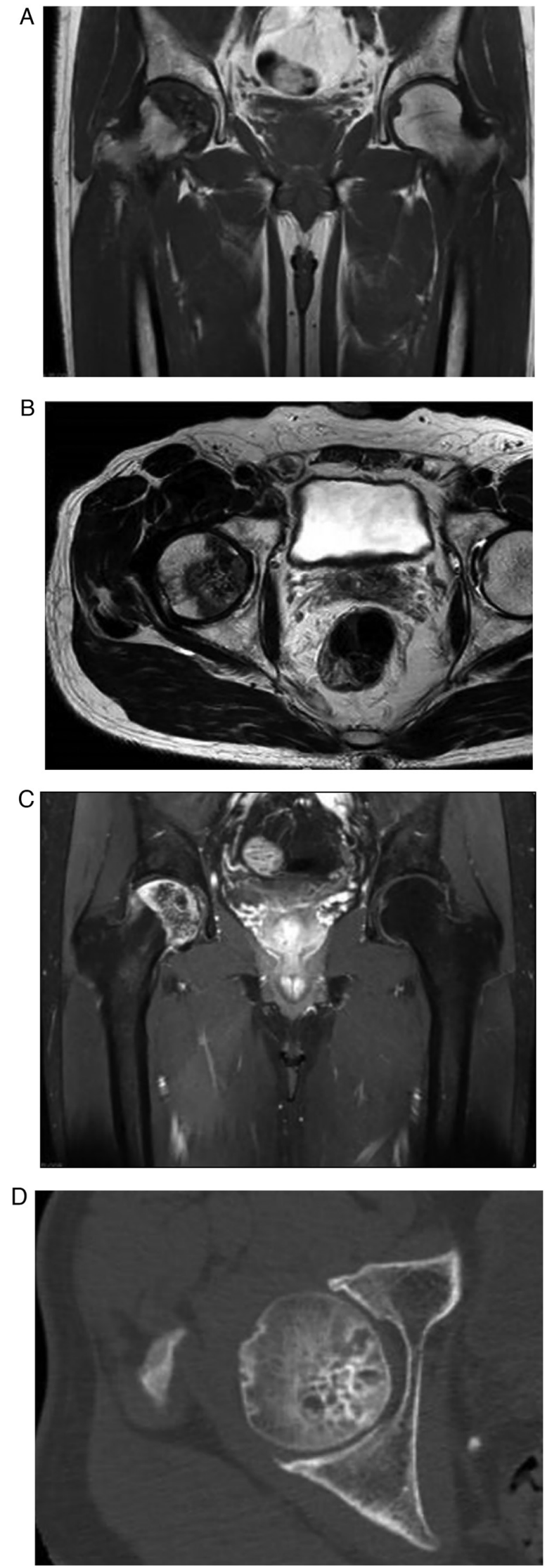

Figure 2. (A) Magnetic resonance imaging using coronal T1W1 of the tumour indicated iso signal intensity in the right femoral head. (B) Axial T2WI indicating heterogeneously high signal intensity with a spotted area (C) The tumour was peripherally enhanced with contrast material on a T1 fat-suppressed image with a focally decreased spotted area. (D) Matrix mineralisation with a characteristic chondroid appearance was evident in the right femoral head on computed tomography. 


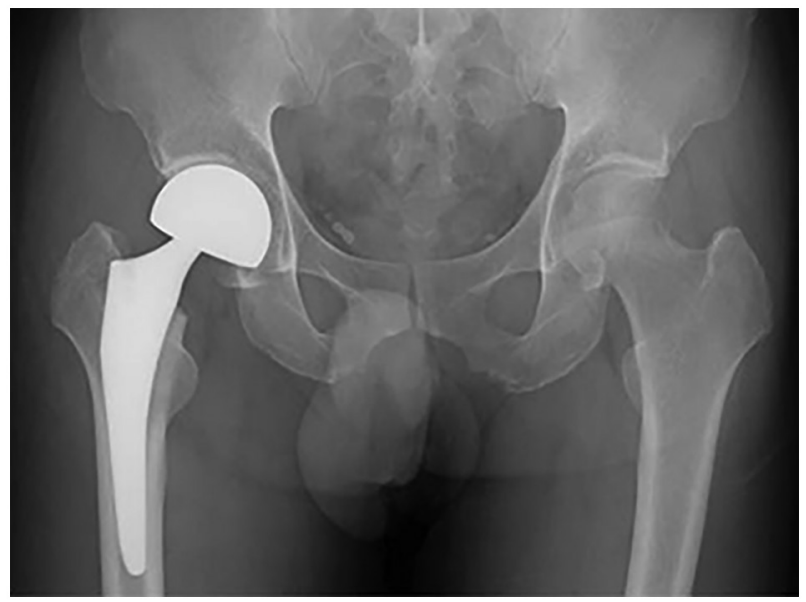

Figure 3. Postoperative plain radiographs displaying the hip hemiarthroplasty performed after en bloc resection.
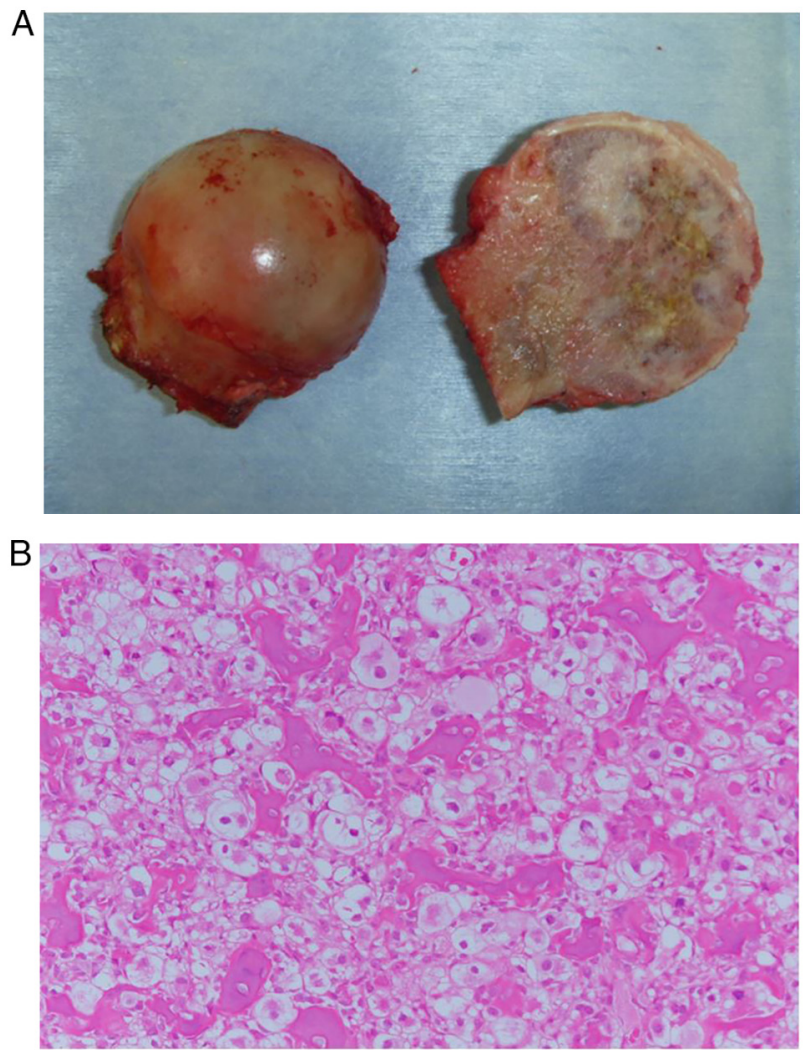

Figure 4. (A) Gross observation indicated that the tumour was composed of chalky-white areas of mineralisation and a chondroid matrix with a well-defined border. The hip joint surface was smooth. (B) Microscopic findings indicated that the tumour cells had a well-defined border and clear cytoplasm. New bone formation was observed. The pathological diagnosis was clear cell chondrosarcoma (H\&E; magnification, x200).

the surgical margin and the postoperative function of the patient, en bloc resection without performing a biopsy may be an acceptable treatment option for radiologically typical CCCS.

Collins et al (11) explored certain typical radiological features of CCCS on plain radiographs, CT scans and MRI, which closely matched those in the present case.
Specifically, the CT scan revealing matrix mineralisation with characteristic chondroid appearance was similar to that in the present case. The World Health Organisation (12) has stated that CCCS is a primary bone tumour primarily affecting the ends of the bone, mainly in the proximal femur and humerus. Radiographically, giant cell tumour of the bone, chondroblastoma, chondrosarcoma and chondroblastic osteosarcoma, and osteonecrosis of the femoral head were all differential diagnoses (3). It is likely that every experienced orthopaedic oncologist in a specialised centre may be capable of suspecting the presence of CCCS in the femoral head, just as in our case.

Histological diagnosis is vital to initiate precise treatment for bone tumours. Compared to an incisional biopsy, a core needle biopsy may at times provide an incorrect diagnosis, usually due to insufficient material quality (6). Therefore, Nakayama et al (5) recommend an incisional biopsy for suspected CCCS over a core needle biopsy. However, incisional biopsy for preoperative diagnosis requires sampling of the tumour, increasing the risk of potentially contaminating healthy tissue.

The reconstruction method after en bloc resection of the femoral head is also worth discussing. Total hip replacement (THR) is able to better maintain the postoperative hip function compared to hemiarthroplasty. However, if the CCCS recurred after THR, particularly in the acetabulum, the second operation would be complicated. Tumour seeding may potentially expand in the overall operating field (8). In the current case, hemiarthroplasty was selected as a prevention of potential tumour expansion in the acetabulum.

CCCS is a low-grade, malignant chondrogenic tumour resistant to chemotherapy and radiotherapy, and surgical removal using adequate margins is the primary treatment strategy for it (4). The surgical margin in these tumours highly correlates with local recurrence and lung metastases (2-5). Itälä et al (2) determined that the 5-year and 10 -year survival rates were 100 and $89 \%$, respectively. Nakayama et al (5) reported that the 5- and 10-year overall survival rates were both $89 \%$. Local recurrence and lung and bone metastases were discovered over five years after the initial diagnosis $(2,3,5)$. Orthopaedic oncologists must be aware that careful long-term follow-up is necessary to detect any recurrences or metastases over time.

In conclusion, in the presence of typical radiological findings for CCCS, en bloc resection without any prior biopsy may be an acceptable treatment for CCCS located on the femoral head.

\section{Acknowledgements}

Not applicable.

\section{Funding}

No funding was received.

\section{Availability of data and materials}

All data generated or analyzed during this study are included in this published article. 


\section{Authors' contributions}

$\mathrm{MH}$ and $\mathrm{HN}$ were responsible for study design and writing of the manuscript. NO and YO analyzed/interpreted the data. AT was responsible for collection of data and scanning images from medical records and performed the histological examination of the tumour. All authors read and approved the final manuscript. NO and YO checked and approved the authenticity of the raw data.

\section{Ethics approval and consent to participate}

Not applicable.

\section{Patient consent for publication}

Written informed consent was obtained from the patient for publication of this case report and any accompanying images.

\section{Competing interests}

The authors declare that they have no competing interests.

\section{References}

1. Unni KK, Dahlin DC, Beabout JW and Sim FH: Chondrosarcoma: Clear-cell variant. A report of sixteen cases. J Bone Joint Surg Am 58: 676-683, 1976.

2. Itälä A, Leerapun T, Inwards C, Collins M and Scully SP: An institutional review of clear cell chondrosarcoma. Clin Orthop Relat Res 440: 209-212, 2005.

3. Donati D, Yin JQ, Colangeli M, Colangeli S, Bella CD, Bacchini P and Bertoni F: Clear cell chondrosarcoma of bone: Long time follow-up of 18 cases. Arch Orthop Trauma Surg 128: 137-142, 2008.
4. Bjornsson J, Unni KK, Dahlin DC, Beabout JW and Sim FH: Clear cell chondrosarcoma of bone. Observations in 47 cases. Am J Surg Pathol 8: 223-230, 1984.

5. Nakayama R, Hayakawa K, Kobayashi E, Endo M, Asano N, Yonemoto T, Kawashima H, Hamada K, Watanabe I, Futani $\mathrm{H}$, et al: What factors are associated with treatment outcomes of Japanese patients with clear cell chondrosarcoma? Clin Orthop Relat Res 478: 2537-2547, 2020.

6. Traina F, Errani C, Toscano A, Pungetti C, Fabbri D, Mazzotti A, Donati D and Faldini C: Current concepts in the biopsy of musculoskeletal tumors. J Bone Joint Surg Am 97: e7, 2015.

7. Berger RA: Mini-incision total hip replacement using an anterolateral approach: Technique and results. Orthop Clin North Am 35: 143-151, 2004.

8. Kawaguchi N, Ahmed AR, Matsumoto S, Manabe J and Matsushita Y: The concept of curative margin in surgery for bone and soft tissue sarcoma. Clin Orthop Relat Res 419: 165-172, 2004.

9. Enneking WF, Dunham W, Gebhardt MC, Malawar M and Pritchard DJ: A system for the functional evaluation of reconstructive procedures after surgical treatment of tumors of the musculoskeletal system. Clin Orthop Relat Res 286: 241-246, 1993.

10. Liu PT, Valadez SD, Chivers FS, Roberts CC and Beauchamp CP: Anatomically based guidelines for core needle biopsy of bone tumors: Implications for limb-sparing surgery. Radiographics 27 : 189-206, 2007.

11. Collins MS, Koyama T, Swee RG and Inwards CY: Clear cell chondrosarcoma: Radiographic, computed tomographic, and magnetic resonance findings in 34 patients with pathologic correlation. Skeletal Radiol 32: 687-694, 2003.

12. Baumhoer D, Bloem JL and Oda Y: Clear cell chondrosarcoma. In: WHO Classification of Tumours of Soft Tissue and Bone. WHO classification of tumours editorial board (eds). IARC Press, Lyon, pp383-384, 2020.

This work is licensed under a Creative Commons Attribution-NonCommercial-NoDerivatives 4.0 International (CC BY-NC-ND 4.0) License. 